\title{
Multi-Term Fractional Differential Equations with Generalized Integral Boundary Conditions
}

\author{
Bashir Ahmad ${ }^{1}\left(\mathbb{D}\right.$, Madeaha Alghanmi ${ }^{1}$, Ahmed Alsaedi ${ }^{1}(\mathbb{D})$ and Sotiris K. Ntouyas ${ }^{1,2, *(D)}$ \\ 1 Nonlinear Analysis and Applied Mathematics (NAAM)-Research Group, Department of Mathematics, \\ Faculty of Science, King Abdulaziz University, P.O. Box 80203, Jeddah 21589, Saudi Arabia \\ 2 Department of Mathematics, University of Ioannina, 45110 Ioannina, Greece \\ * Correspondence: sntouyas@uoi.gr
}

Received: 23 July 2019; Accepted: 15 August 2019; Published: 18 August 2019

Abstract: We discuss the existence of solutions for a Caputo type multi-term nonlinear fractional differential equation supplemented with generalized integral boundary conditions. The modern tools of functional analysis are applied to achieve the desired results. Examples are constructed for illustrating the obtained work. Some new results follow as spacial cases of the ones reported in this paper.

Keywords: Caputo fractional derivative; multi-order fractional differential equations; generalized fractional integral; existence; fixed point

\section{Introduction}

Fractional calculus received overwhelming interest in view of its vast applications in the mathematical modeling of real world phenomena occurring in scientific and engineering disciplines. Fractional-order operators give rise to more realistic models than the ones relying on classical calculus due to their nonlocal nature, which takes into account the past effects of the phenomena under consideration. Applications of fractional calculus appear in numerous fields such as continuum mechanics [1], bioengineering [2], physics [3], financial and economy dynamics [4], disease models [5], ecology [6], etc. For further details, one can see the texts [7-10].

Fractional-order initial and boundary value problems, involving different kinds of derivatives such as Riemann-Liouville, Caputo, and Hadamard type, have been studied by many authors. The literature on the topic is rapidly augmented with a variety of interesting and useful results during the past few years (see, for instance, [11-26] and references therein).

In this paper, we introduce and study a nonlinear nonlocal-terminal value problem consisting of multiple Caputo fractional derivatives and generalized integral boundary conditions. In precise terms, we investigate the following problem:

$$
\left\{\begin{array}{l}
{ }^{c} D^{\alpha}\left[{ }^{c} D^{\beta} y(t)-g(t, y(t))\right]=f(t, y(t)), t \in[0, T], \\
a_{1} y(\eta)+b_{1} y(T)=c \\
a_{2}{ }^{c} D^{p} y(\eta)+b_{2}{ }^{c} D^{p} y(T)=\sum_{i=1}^{n} \lambda_{i} \rho_{i} I_{\theta_{i}, \kappa_{i}}^{\gamma_{i}, \delta_{i}} y\left(\xi_{i}\right), \eta, \xi_{i} \in(0, T),
\end{array}\right.
$$

where ${ }^{c} D^{\chi}$ denotes the Caputo fractional derivatives of order $\chi \in\{\alpha, \beta, p\}$ with $0<\chi<1, p<\beta$, $\rho_{i} I_{\theta_{i}, \kappa_{i}}^{\gamma_{i}, \delta_{i}}$ is the generalized fractional integral, $\gamma_{i}>0, \delta_{i}, \theta_{i}, \kappa_{i}, \lambda_{i} \in \mathbb{R}, i=1,2, \ldots, n, a_{i}, b_{i} \in \mathbb{R}, i=1,2$ and $f, g:[0, T] \times \mathbb{R} \rightarrow \mathbb{R}$ are given continuous functions.

Here, it is imperative to note that the generalized integral boundary conditions [27] introduced in the problem in Equation (1) are quite general in nature and lead to Riemann-Liouville, Hadamard, 
Erdélyi-Kober and Katugampola type conditions for particular values of the parameters in these conditions (see Remark 1).

The rest of the paper is organized as follows. In Section 2, we recall some basic concepts of fractional calculus and prove a lemma relating the problem in Equation (1) with an integral equation, while the main results, relying on Krasnoselskii fixed point theorem, Leray-Schauder nonlinear alternative and Banach contraction mapping principle, are presented in Section 3. Section 4 contains illustrative examples for the obtained results. In Section 5 , we deduce some new results by fixing the the parameters in the derived results.

\section{Preliminary Concepts and Integral Solution}

Let us begin this section with some basic definitions [7].

Definition 1. The Riemann-Liouville fractional integral of a locally integrable real-valued function $\mu$ of order $\tau \in \mathbb{R}(\tau>0)$ on $-\infty \leq a_{1}<t<a_{2} \leq+\infty$, denoted by $J_{a_{1}}^{\tau}$ is defined by

$$
J_{a_{1}}^{\tau} \mu(t)=\frac{1}{\Gamma(\alpha)} \int_{a_{1}}^{t}(t-s)^{\tau-1} \mu(s) d s,
$$

where $\Gamma$ denotes the Euler gamma function.

Definition 2. If $\mu \in C^{m}\left[a_{1}, a_{2}\right]$, then the Caputo fractional derivative ${ }^{c} D_{a_{1}}^{\tau}$ of order $\tau \in \mathbb{R}(m-1<\tau<$ $m, m \in \mathbb{N}$ ) of $\mu$ is defined as

$$
{ }^{c} D_{a_{1}}^{\tau} \mu(t)=\frac{1}{\Gamma(m-\tau)} \int_{a_{1}}^{t}(t-s)^{m-1-\tau} \mu^{(m)}(s) d s .
$$

In the sequel, we represent Riemann-Liouville fractional integral and Caputo fractional derivative by $J^{\tau}$ and ${ }^{c} D^{\tau}$, respectively, when $a_{1}=0$.

Lemma 1. With the given notations, the following equality holds:

$$
J^{\tau}\left({ }^{c} D^{\tau} h(u)\right)=h(u)-c_{0}-c_{1} u-\cdots-c_{n-1} u^{n-1}, u>0, n-1<\tau<n,
$$

where $c_{i}(i=0,1, \ldots, n-1)$ are arbitrary constants [7].

For $1 \leq p \leq \infty, c \in \mathbb{R}$, we denote by $X_{c}^{p}\left(a_{1}, a_{2}\right)$ the space of all complex-valued Lebesgue measurable functions $\psi$ on $\left(a_{1}, a_{2}\right)$ with

$$
\|\psi\|_{X_{c}^{p}}=\left(\int_{a_{1}}^{a_{2}}\left|x^{c} \psi(x)\right|^{p} \frac{d x}{x}\right)^{1 / p}<\infty .
$$

Definition 3 ([27]). Let $f \in X_{c}^{p}\left(a_{1}, a_{2}\right), \alpha, \beta>0$ and $\rho, \eta, \kappa \in \mathbb{R}$. The generalized fractional integral is defined by

$$
\left({ }^{\rho} I_{\eta, \kappa}^{\alpha, \beta} f\right)(t)=\frac{\rho^{1-\beta} t^{\kappa}}{\Gamma(\alpha)} \int_{a_{1}}^{t} \frac{s^{\rho(\eta+1)-1}}{\left(t^{\rho}-s^{\rho}\right)^{1-\alpha}} f(s) d s, \quad a_{1} \leq t<a_{2} \leq \infty,
$$

if the integral exists. 
Letting $f(t)=t^{q}, a_{1}=0$ in the above definition, we obtain the formula:

$$
{ }^{\rho} I_{\eta, \kappa}^{\alpha, \beta} t^{q}=\frac{t^{\kappa+\rho(\eta+\alpha)+q}}{\rho^{\beta}} \frac{\Gamma\left(\eta+\frac{q}{\rho}+1\right)}{\Gamma\left(\eta+\frac{q}{\rho}+\alpha+1\right)} .
$$

Remark 1. The integral operator in Equation (3) reduces to: (i) Riemann-Liouville fractional integral for $\kappa=0, \eta=0$ and $\rho \rightarrow 1$; (ii) Hadamard fractional integral for $\beta=\alpha, \kappa=0, \eta=0$, and $\rho \rightarrow 0+$; (iii) Erdélyi-Kober fractional integral for $\beta=0$ and $\kappa=-\rho(\alpha+\eta)$; and (iv) Katugampola fractional integral for $\beta=\alpha, \kappa=0$ and $\eta=0$. For more details, see [27].

In the sequel, we set the following notations for the sake of computational convenience.

$$
\begin{aligned}
A_{1} & =\frac{a_{1} \eta^{\beta}+b_{1} T^{\beta}}{\Gamma(\beta+1)} \\
A_{2} & =\frac{\left(a_{2} \eta^{\beta-p}+b_{2} T^{\beta-p}\right)}{\Gamma(1+\beta-p)}-\sum_{i=1}^{n} \lambda_{i} \frac{\Gamma\left(\theta_{i}+\frac{\beta}{\rho_{i}}+1\right) \xi_{i}^{\kappa_{i}+\rho_{i}\left(\theta_{i}+\gamma_{i}\right)+\beta}}{\Gamma(\beta+1) \Gamma\left(\theta_{i}+\frac{\beta}{\rho_{i}}+\gamma_{i}+1\right) \rho_{i}^{\delta_{i}}}, \\
B_{1} & =a_{1}+b_{1} \\
B_{2} & =\sum_{i=1}^{n} \lambda_{i} \frac{\Gamma\left(\theta_{i}+1\right) \xi_{i}^{\kappa_{i}+\rho_{i}\left(\theta_{i}+\gamma_{i}\right)}}{\Gamma\left(\theta_{i}+\gamma_{i}+1\right) \rho_{i}^{\delta_{i}}} \\
\Omega & =-\left(A_{1} B_{2}+B_{1} A_{2}\right) .
\end{aligned}
$$

Lemma 2. Let $\Omega \neq 0$. Then, the function $y$ is a solution of the problem

$$
\left\{\begin{array}{l}
{ }^{c} D^{\alpha}\left[{ }^{c} D^{\beta} y(t)-g(t, y(t))\right]=f(t, y(t)), t \in[0, T], \\
a_{1} y(\eta)+b_{1} y(T)=c \\
a_{2}{ }^{c} D^{p} y(\eta)+b_{2}{ }^{c} D^{p} y(T)=\sum_{i=1}^{n} \lambda_{i}{ }^{\rho_{i}} I_{\theta_{i}, \kappa_{i}}^{\gamma_{i}, \delta_{i}} y\left(\xi_{i}\right), \eta, \xi_{i} \in(0, T),
\end{array}\right.
$$

if and only if

$$
\begin{aligned}
y(t)= & J^{\beta} g(t, y(t))+J^{\alpha+\beta} f(t, y(t))+\frac{t^{\beta}}{\Omega \Gamma(\beta+1)}\left\{-B_{2}\left[c-a_{1} J^{\beta} g(\eta, y(\eta))\right.\right. \\
& \left.-b_{1} J^{\beta} g(T, y(T))-a_{1} J^{\alpha+\beta} f(\eta, y(\eta))-b_{1} J^{\alpha+\beta} f(T, y(T))\right] \\
& -B_{1}\left[\sum_{i=1}^{n} \lambda_{i} \rho_{i} I_{\theta_{i}, \kappa_{i}}^{\gamma_{i} \delta_{i}}\left(J^{\beta} g\left(\xi_{i}, y\left(\xi_{i}\right)\right)+J^{\alpha+\beta} f\left(\xi_{i}, y\left(\xi_{i}\right)\right)\right)-a_{2} J^{\beta-p} g(\eta, y(\eta))\right. \\
& \left.\left.-b_{2} J^{\beta-p} g(T, y(T))-a_{2} J^{\alpha+\beta-p} f(\eta, y(\eta))-b_{2} J^{\alpha+\beta-p} f(T, y(T))\right]\right\} \\
& +\frac{1}{\Omega}\left\{A _ { 1 } \left[\sum_{i=1}^{n} \lambda_{i} \rho_{i} I_{\theta_{i}, \kappa_{i}}^{\gamma_{i} \delta_{i}}\left(J^{\beta} g\left(\xi_{i}, y\left(\xi_{i}\right)\right)+J^{\alpha+\beta} f\left(\xi_{i}, y\left(\xi_{i}\right)\right)\right)-a_{2} J^{\beta-p} g(\eta, y(\eta))\right.\right. \\
& \left.-b_{2} J^{\beta-p} g(T, y(T))-a_{2} J^{\alpha+\beta-p} f(\eta, y(\eta))-b_{2} J^{\alpha+\beta-p} f(T, y(T))\right] \\
& -A_{2}\left[c-a_{1} J^{\beta} g(\eta, y(\eta))-b_{1} J^{\beta} g(T, y(T))-a_{1} J^{\alpha+\beta} f(\eta, y(\eta))\right. \\
& \left.\left.-b_{1} J^{\alpha+\beta} f(T, y(T))\right]\right\} .
\end{aligned}
$$

Proof. Applying the operator $J^{\alpha}$ on both sides of the fractional differential equation in Equation (10), we get

$$
D^{\beta} y(t)-g(t, y(t))=J^{\alpha} f(t, y(t))+c_{1}
$$


where $c_{1} \in \mathbb{R}$ is an arbitrary constant and moreover

$$
y(t)=J^{\beta} g(t, y(t))+J^{\alpha+\beta} f(t, y(t))+\frac{t^{\beta}}{\Gamma(\beta+1)} c_{1}+c_{2},
$$

where $c_{2} \in \mathbb{R}$ is an arbitrary constant. Taking Riemann-Liouville fractional derivative and the generalized fractional integral of order $\delta_{i}, \gamma_{i}>0$ of Equation (12), respectively, yields

$$
\begin{gathered}
{ }^{c} D^{p} y(t)=J^{\beta-p} g(t, y(t))+J^{\alpha+\beta-p} f(t, y(t))+\frac{t^{\beta-p}}{\Gamma(\beta-p+1)} c_{1}, \\
\rho_{i}{ }_{\theta_{i}, \kappa_{i}}^{\gamma_{i}, \delta_{i}} y(t)= \\
\quad{ }^{\rho_{i}} I_{\theta_{i}, \kappa_{i}}^{\gamma_{i}, \delta_{i}}\left(J^{\beta} g(t, y(t))+J^{\alpha+\beta} f(t, y(t))\right) \\
\quad+c_{1} \frac{1}{\Gamma(1+\beta)} \frac{t^{\kappa_{i}+\rho_{i}\left(\theta_{i}+\gamma_{i}\right)+\beta}}{\rho_{i}^{\delta_{i}}} \frac{\Gamma\left(\theta_{i}+\frac{\beta}{\rho_{i}}+1\right)}{\Gamma\left(\theta_{i}+\frac{\beta}{\rho_{i}}+\gamma_{i}+1\right)}+c_{2} \frac{\Gamma\left(\theta_{i}+1\right) t^{\kappa_{i}+\rho_{i}\left(\theta_{i}+\gamma_{i}\right)}}{\Gamma\left(\theta_{i}+\gamma_{i}+1\right) \rho_{i}^{\delta_{i}}} .
\end{gathered}
$$

Using the boundary conditions given by Equation (10) together with Equations (12)-(14), we obtain

$$
\begin{gathered}
A_{1} c_{1}+B_{1} c_{2}=c-a_{1} J^{\beta} g(\eta, y(\eta))-b_{1} J^{\beta} g(T, y(T)) \\
-a_{1} J^{\alpha+\beta} f(\eta, y(\eta))-b_{1} J^{\alpha+\beta} f(T, y(T)), \\
A_{2} c_{1}-B_{2} c_{2}=\sum_{i=1}^{n} \lambda_{i}{ }^{\rho_{i}} I_{\theta_{i}, \kappa_{i}}^{\gamma_{i}, \delta_{i}}\left(J^{\beta} g\left(\xi_{i}, y\left(\xi_{i}\right)\right)+J^{\alpha+\beta} f\left(\xi_{i}, y\left(\xi_{i}\right)\right)\right) \\
-a_{2} J^{\beta-p} g(\eta, y(\eta))-a_{2} J^{\alpha+\beta-p} f(\eta, y(\eta)) \\
-b_{2} J^{\beta-p} g(T, y(T))-b_{2} J^{\alpha+\beta-p} f(T, y(T)),
\end{gathered}
$$

where $A_{i}, B_{i}, i=1,2$ are given by Equations (5)-(8). Solving the system of Equations (15) and (16) for $c_{1}$ and $c_{2}$, we find that

$$
\begin{aligned}
c_{1}= & \frac{1}{\Omega}\left[-B_{2}\left\{c-a_{1} J^{\beta} g(\eta, y(\eta))-b_{1} J^{\beta} g(T, y(T))-a_{1} J^{\alpha+\beta} f(\eta, y(\eta))-b_{1} J^{\alpha+\beta} f(T, y(T))\right\}\right. \\
& -B_{1}\left\{\sum_{i=1}^{n} \lambda_{i}{ }^{\rho_{i}} I_{\theta_{i}, \kappa_{i}}^{\gamma_{i}, \delta_{i}}\left(J^{\beta} g\left(\xi_{i}, y\left(\xi_{i}\right)\right)+J^{\alpha+\beta} f\left(\xi_{i}, y\left(\xi_{i}\right)\right)\right)\right. \\
& \left.\left.-a_{2} J^{\beta-p} g(\eta, y(\eta))-b_{2} J^{\beta-p} g(T, y(T))-a_{2} J^{\alpha+\beta-p} f(\eta, y(\eta))-b_{2} J^{\alpha+\beta-p} f(T, y(T))\right\}\right]
\end{aligned}
$$

and

$$
\begin{aligned}
c_{2}= & \frac{1}{\Omega}\left[A _ { 1 } \left\{\sum_{i=1}^{n} \lambda_{i}{ }^{\rho_{i}} I_{\theta_{i}, \kappa_{i}}^{\gamma_{i}, \delta_{i}}\left(J^{\beta} g\left(\xi_{i}, y\left(\xi_{i}\right)\right)+J^{\alpha+\beta} f\left(\xi_{i}, y\left(\xi_{i}\right)\right)\right)\right.\right. \\
& \left.-a_{2} J^{\beta-p} g(\eta, y(\eta))-b_{2} J^{\beta-p} g(T, y(T))-a_{2} J^{\alpha+\beta-p} f(\eta, y(\eta))-b_{2} J^{\alpha+\beta-p} f(T, y(T))\right\} \\
& \left.-A_{2}\left\{c-a_{1} J^{\beta} g(\eta, y(\eta))-b_{1} J^{\beta} g(T, y(T))-a_{1} J^{\alpha+\beta} f(\eta, y(\eta))-b_{1} J^{\alpha+\beta} f(T, y(T))\right\}\right] .
\end{aligned}
$$

Substituting the values of $c_{1}$ and $c_{2}$ in Equation (12), we obtain the solution in Equation (11). By direct computation, it is easy to accomplish the converse of the lemma. The proof is complete. 


\section{Existence and Uniqueness Results}

Using Lemma 2, we define an operator $F: C([0, T], \mathbb{R}) \rightarrow C([0, T], \mathbb{R})$ by

$$
\begin{aligned}
F y(t)= & J^{\beta} g(t, y(t))+J^{\alpha+\beta} f(t, y(t))+\frac{t^{\beta}}{\Omega \Gamma(\beta+1)}\left\{-B_{2}\left[c-a_{1} J^{\beta} g(\eta, y(\eta))\right.\right. \\
& \left.-b_{1} J^{\beta} g(T, y(T))-a_{1} J^{\alpha+\beta} f(\eta, y(\eta))-b_{1} J^{\alpha+\beta} f(T, y(T))\right] \\
& -B_{1}\left[\sum_{i=1}^{n} \lambda_{i} \rho_{i} I_{\theta_{i}, \kappa_{i}}^{\gamma_{i}, \delta_{i}}\left(J^{\beta} g\left(\xi_{i}, y\left(\xi_{i}\right)\right)+J^{\alpha+\beta} f\left(\xi_{i}, y\left(\xi_{i}\right)\right)\right)-a_{2} J^{\beta-p} g(\eta, y(\eta))\right. \\
& \left.\left.-b_{2} J^{\beta-p} g(T, y(T))-a_{2} J^{\alpha+\beta-p} f(\eta, y(\eta))-b_{2} J^{\alpha+\beta-p} f(T, y(T))\right]\right\} \\
& +\frac{1}{\Omega}\left\{A _ { 1 } \left[\sum_{i=1}^{n} \lambda_{i} \rho_{i} I_{\theta_{i}, \kappa_{i}}^{\gamma_{i}, \delta_{i}}\left(J^{\beta} g\left(\xi_{i}, y\left(\xi_{i}\right)\right)+J^{\alpha+\beta} f\left(\xi_{i}, y\left(\xi_{i}\right)\right)\right)-a_{2} I^{\beta-p} g(\eta, y(\eta))\right.\right. \\
& \left.-b_{2} J^{\beta-p_{g}}(T, y(T))-a_{2} J^{\alpha+\beta-p} f(\eta, y(\eta))-b_{2} J^{\alpha+\beta-p} f(T, y(T))\right] \\
& -A_{2}\left[c-a_{1} J^{\beta} g(\eta, y(\eta))-b_{1} J^{\beta} g(T, y(T))-a_{1} J^{\alpha+\beta} f(\eta, y(\eta))\right. \\
& \left.\left.-b_{1} J^{\alpha+\beta} f(T, y(T))\right]\right\},
\end{aligned}
$$

where $C([0, T], \mathbb{R})$ denotes the Banach space of all continuous functions from $[0, T]$ to $\mathbb{R}$ endowed with the sup-norm.

In the forthcoming analysis, we write $\mathcal{C}=C([0, T], \mathbb{R})$ and use the notations:

$$
\begin{aligned}
\Lambda_{1}= & \frac{T^{\beta}}{\Gamma(\beta+1)}+\frac{T^{\beta}}{|\Omega| \Gamma(\beta+1)}\left\{\left|B_{2}\right| \frac{\left|a_{1}\right| \eta^{\beta}+\left|b_{1}\right| T^{\beta}}{\Gamma(\beta+1)}\right. \\
& \left.+\left|B_{1}\right|\left[\sum_{i=1}^{n}\left|\lambda_{i}\right| \frac{\xi_{i}^{\kappa_{i}+\rho_{i}\left(\theta_{i}+\gamma_{i}\right)+\beta} \Gamma\left(\theta_{i}+\frac{\beta}{\rho_{i}}+1\right)}{\rho_{i}^{\delta_{i}} \Gamma(\beta+1) \Gamma\left(\gamma_{i}+\theta_{i}+\frac{\beta}{\rho_{i}}+1\right)}+\frac{\left|a_{2}\right| \eta^{\beta-p}+\left|b_{2}\right| T^{\beta-p}}{\Gamma(\beta-p+1)}\right]\right\} \\
& +\frac{1}{|\Omega|}\left\{\left|A_{1}\right|\left[\sum_{i=1}^{n}\left|\lambda_{i}\right| \frac{\xi_{i}^{\kappa_{i}+\rho_{i}\left(\theta_{i}+\gamma_{i}\right)+\beta} \Gamma\left(\theta_{i}+\frac{\beta}{\rho_{i}}+1\right)}{\rho_{i}^{\delta_{i}} \Gamma(\beta+1) \Gamma\left(\gamma_{i}+\theta_{i}+\frac{\beta}{\rho_{i}}+1\right)}+\frac{\left|a_{2}\right| \eta^{\beta-p}+\left|b_{2}\right| T^{\beta-p}}{\Gamma(\beta-p+1)}\right]\right. \\
& \left.+\left|A_{2}\right| \frac{\left|a_{1}\right| \eta^{\beta}+\left|b_{1}\right| T^{\beta}}{\Gamma(\beta+1)}\right\}
\end{aligned}
$$

and

$$
\begin{aligned}
\Lambda_{2}= & \frac{T^{\alpha+\beta}}{\Gamma(\alpha+\beta+1)}+\frac{T^{\beta}}{|\Omega| \Gamma(\beta+1)}\left\{\left|B_{2}\right| \frac{\left|a_{1}\right| \eta^{\alpha+\beta}+\left|b_{1}\right| T^{\alpha+\beta}}{\Gamma(\alpha+\beta+1)}\right. \\
& \left.+\left|B_{1}\right|\left[\sum_{i=1}^{n}\left|\lambda_{i}\right| \frac{\xi_{i}^{\kappa_{i}+\rho_{i}\left(\theta_{i}+\gamma_{i}\right)+\alpha+\beta} \Gamma\left(\theta_{i}+\frac{\alpha+\beta}{\rho_{i}}+1\right)}{\rho_{i}^{\delta_{i}} \Gamma(\alpha+\beta+1) \Gamma\left(\gamma_{i}+\theta_{i}+\frac{\alpha+\beta}{\rho_{i}}+1\right)}+\frac{\left|a_{2}\right| \eta^{\alpha+\beta-p}+\left|b_{2}\right| T^{\alpha+\beta-p}}{\Gamma(\alpha+\beta-p+1)}\right]\right\} \\
& +\frac{1}{|\Omega|}\left\{\left|A_{1}\right|\left[\sum_{i=1}^{n}\left|\lambda_{i}\right| \frac{\xi_{i}^{\kappa_{i}+\rho_{i}\left(\theta_{i}+\gamma_{i}\right)+\alpha+\beta} \Gamma\left(\theta_{i}+\frac{\alpha+\beta}{\rho_{i}}+1\right)}{\rho_{i}^{\delta_{i}} \Gamma(\alpha+\beta+1) \Gamma\left(\gamma_{i}+\theta_{i}+\frac{\alpha+\beta}{\rho_{i}}+1\right)}+\frac{\left|a_{2}\right| \eta^{\alpha+\beta-p}+\left|b_{2}\right| T^{\alpha+\beta-p}}{\Gamma(\alpha+\beta-p+1)}\right]\right. \\
& \left.+\left|A_{2}\right| \frac{\left|a_{1}\right| \eta^{\alpha+\beta}+\left|b_{1}\right| T^{\alpha+\beta}}{\Gamma(\alpha+\beta+1)}\right\} .
\end{aligned}
$$

\subsection{Existence Result via Kransnoselskii's Fixed Point Theorem}

In the following existence result, we apply Krasnoselskii fixed point theorem [28].

Theorem 1. Let the following assumptions hold: 
$\left(G_{1}\right) \quad f, g:[0, T] \times \mathbb{R} \rightarrow \mathbb{R}$ are continuous functions.

$\left(G_{2}\right)$ There exists a nonnegative constant $k$ such that

$$
|g(t, y)-g(t, x)| \leq k\|y-x\|, \quad \text { for } t \in[0, T] \text { and every } y, x \in \mathbb{R} .
$$

$\left(G_{3}\right)$ There exist continuous nonnegative functions $\psi, \phi \in C\left([0, T], \mathbb{R}^{+}\right)$such that

$$
|g(t, y)| \leq \psi(t), \quad|f(t, y)| \leq \phi(t), \quad \forall(t, y) \in[0, T] \times \mathbb{R}
$$

Then, the BVP in Equation (1) has at least one solution on [0,T], provided

$$
k \Lambda_{1}<1
$$

where $\Lambda_{1}$ is given by Equation (18).

Proof. By Assumption $\left(G_{3}\right)$, we can fix

$$
\rho \geq|| \psi|| \Lambda_{1}+|| \phi|| \Lambda_{2}+\frac{|c|}{|\Omega|}\left[\left|B_{2}\right| \frac{T^{\beta}}{\Gamma(\beta+1)}+\left|A_{2}\right|\right],
$$

where $\sup _{t \in[0, T]}|\psi(t)|=\|\psi\|, \sup _{t \in[0, T]}|\phi(t)|=\|\phi\|$ and consider $B_{\rho}=\{y \in C([0, T], \mathbb{R}):\|y\| \leq \rho\}$. Let us split the operator $F: C([0, T], \mathbb{R}) \rightarrow C([0, T], \mathbb{R})$ defined by Equation (17) as $F=F_{1}+F_{2}$, where $F_{1}$ and $F_{2}$ are given by

$$
\begin{aligned}
F_{1}(y)(t)= & J^{\beta} g(t, y(t))+\frac{t^{\beta}}{\Omega \Gamma(\beta+1)}\left\{-B_{2}\left[c-a_{1} J^{\beta} g(\eta, y(\eta))-b_{1} I^{\beta} g(T, y(T))\right]\right. \\
& \left.-B_{1}\left[\sum_{i=1}^{n} \lambda_{i} \rho_{i} I_{\theta_{i}, \kappa_{i}}^{\gamma_{i}, \delta_{i}}\left(J^{\beta} g\left(\xi_{i}, y\left(\xi_{i}\right)\right)\right)-a_{2} J^{\beta-p} g(\eta, y(\eta))-b_{2} J^{\beta-p} g(T, y(T))\right]\right\} \\
& +\frac{1}{\Omega}\left\{A_{1}\left[\sum_{i=1}^{n} \lambda_{i} \rho_{i} I_{\theta_{i}, \kappa_{i}}^{\gamma_{i}, \delta_{i}}\left(J^{\beta} g\left(\xi_{i}, y\left(\xi_{i}\right)\right)\right)-a_{2} J^{\beta-p} g(\eta, y(\eta))-b_{2} J^{\beta-p} g(T, y(T))\right]\right. \\
& \left.-A_{2}\left[c-a_{1} J^{\beta} g(\eta, y(\eta))-b_{1} J^{\beta} g(T, y(T))\right]\right\},
\end{aligned}
$$

and

$$
\begin{aligned}
F_{2}(y)(t)= & J^{\alpha+\beta} f(t, y(t))+\frac{t^{\beta}}{\Omega \Gamma(\beta+1)}\left\{-B_{2}\left[-a_{1} J^{\alpha+\beta} f(\eta, y(\eta))-b_{1} J^{\alpha+\beta} f(T, y(T))\right]\right. \\
& \left.-B_{1}\left[\sum_{i=1}^{n} \lambda_{i} \rho_{i} I_{\theta_{i}, \kappa_{i}}^{\gamma_{i} \delta_{i}}\left(J^{\alpha+\beta} f\left(\xi_{i}, y\left(\xi_{i}\right)\right)\right)-a_{2} J^{\alpha+\beta-p} f(\eta, y(\eta))-b_{2} J^{\alpha+\beta-p} f(T, y(T))\right]\right\} \\
& +\frac{1}{\Omega}\left\{A_{1}\left[\sum_{i=1}^{n} \lambda_{i} \rho_{i} I_{\theta_{i}, \kappa_{i}}^{\gamma_{i}, \delta_{i}}\left(J^{\alpha+\beta} f\left(\xi_{i}, y\left(\xi_{i}\right)\right)\right)-a_{2} J^{\alpha+\beta-p} f(\eta, y(\eta))-b_{2} J^{\alpha+\beta-p} f(T, y(T))\right]\right. \\
& \left.-A_{2}\left[-a_{1} J^{\alpha+\beta} f(\eta, y(\eta))-b_{1} J^{\alpha+\beta} f(T, y(T))\right]\right\} .
\end{aligned}
$$


For any $x, y \in B_{\rho}$, we have

$$
\begin{aligned}
& \left|F_{1} x(t)+F_{2} y(t)\right| \\
\leq & J^{\beta}|g(t, x(t))|+J^{\alpha+\beta}|f(t, y(t))|+\frac{T^{\beta}}{|\Omega| \Gamma(\beta+1)}\left\{| B _ { 2 } | \left[|c|+\left|a_{1}\right| J^{\beta}|g(\eta, x(\eta))|\right.\right. \\
& \left.+\left|b_{1}\right| J^{\beta}|g(T, x(T))|+\left|a_{1}\right| J^{\alpha+\beta}|f(\eta, y(\eta))|+\left|b_{1}\right| J^{\alpha+\beta}|f(T, y(T))|\right] \\
& +\left|B_{1}\right|\left[\sum_{i=1}^{n}\left|\lambda_{i}\right|{ }^{\rho_{i}} I_{\theta_{i}, \kappa_{i}}^{\gamma_{i}},_{i}\left(J^{\beta}\left|g\left(\xi_{i}, x\left(\xi_{i}\right)\right)\right|+J^{\alpha+\beta}\left|f\left(\xi_{i}, y\left(\xi_{i}\right)\right)\right|\right)+\left|a_{2}\right| J^{\beta-p}|g(\eta, x(\eta))|\right. \\
& \left.\left.+\left|b_{2}\right| J^{\beta-p}|g(T, x(T))|+\left|a_{2}\right| J^{\alpha+\beta-p}|f(\eta, y(\eta))|+\left|b_{2}\right| J^{\alpha+\beta-p}|f(T, y(T))|\right]\right\} \\
& +\frac{1}{|\Omega|}\left\{| A _ { 1 } | \left[\sum_{i=1}^{n}\left|\lambda_{i}\right|{ }^{\rho_{i}} I_{\theta_{i}, \kappa_{i}}^{\gamma_{i}, \delta_{i}}\left(J^{\beta}\left|g\left(\xi_{i}, x\left(\xi_{i}\right)\right)\right|+J^{\alpha+\beta}\left|f\left(\xi_{i}, y\left(\xi_{i}\right)\right)\right|\right)+\left|a_{2}\right| J^{\beta-p}|g(\eta, x(\eta))|\right.\right. \\
& \left.+\left|b_{2}\right| J^{\beta-p}|g(T, x(T))|+\left|a_{2}\right| J^{\alpha+\beta-p}|f(\eta, y(\eta))|+\left|b_{2}\right| J^{\alpha+\beta-p}|f(T, y(T))|\right] \\
& +\left|A_{2}\right|\left[|c|+\left|a_{1}\right| J^{\beta}|g(\eta, x(\eta))|+\left|b_{1}\right| J^{\beta}|g(T, x(T))|+\left|a_{1}\right| J^{\alpha+\beta}|f(\eta, y(\eta))|\right. \\
& \left.\left.+\left|b_{1}\right| J^{\alpha+\beta}|f(T, y(T))|\right]\right\} \\
\leq & \|\psi\| \Lambda_{1}+\|\phi\| \Lambda_{2}+\frac{|c|}{|\Omega|}\left[\left|B_{2}\right| \frac{T^{\beta}}{\Gamma(\beta+1)}+\left|A_{2}\right|\right] \leq \rho .
\end{aligned}
$$

Hence, $\left\|F_{1} x+F_{2} y\right\| \leq \rho$, which shows that $F_{1} x+F_{2} y \in B_{\rho}$. A direct application of the condition in Equation (20) implies that $F_{1}$ is a contraction mapping. In view of the continuity of $f$, it follows that the operator $F_{2}$ is continuous. Since $\left\|F_{2} y\right\| \leq\|\phi\| \Lambda_{2}, F_{2}$ is uniformly bounded on $B_{\rho}$.

Finally, we prove the compactness of the operator $F_{2}$. For that, we define $\sup _{(t, x) \in[0, T] \times B_{\rho}}|f(t, y)|=\bar{f}<\infty$ and take $t_{1}, t_{2} \in[0, T], t_{1}<t_{2}$. Then,

$$
\begin{aligned}
& \left|F_{2} y\left(t_{2}\right)-F_{2} y\left(t_{1}\right)\right| \\
\leq & \frac{\bar{f}}{\Gamma(\alpha+\beta+1)}\left[t_{2}^{\alpha+\beta}-t_{1}^{\alpha+\beta}+2\left(t_{2}-t_{1}\right)^{\alpha+\beta}\right] \\
& +\frac{\left|t_{2}^{\beta}-t_{1}^{\beta}\right| \bar{f}}{|\Omega| \Gamma(\beta+1)}\left\{\left|B_{2}\right| \frac{\left|a_{1}\right| \eta^{\alpha+\beta}+\left|b_{1}\right| T^{\alpha+\beta}}{\Gamma(\alpha+\beta+1)}+\left|B_{1}\right|\left[\sum_{i=1}^{n}\left|\lambda_{i}\right| \frac{\xi_{i}^{\kappa_{i}+\rho_{i}\left(\theta_{i}+\gamma_{i}\right)+\alpha+\beta} \Gamma\left(\theta_{i}+\frac{\alpha+\beta}{\rho_{i}}+1\right)}{\rho_{i}^{\delta_{i}} \Gamma(\alpha+\beta+1) \Gamma\left(\gamma_{i}+\theta_{i}+\frac{\alpha+\beta}{\rho_{i}}+1\right)}\right.\right. \\
& \left.\left.+\frac{\left|a_{2}\right| \eta^{\alpha+\beta-p}+\left|b_{2}\right| T^{\alpha+\beta-p}}{\Gamma(\alpha+\beta-p+1)}\right]\right\} \rightarrow 0 \text { as } t_{2}-t_{1} \rightarrow 0,
\end{aligned}
$$

independently of $y \in B_{\rho}$. Thus, $F_{2}$ is equicontinuous. In view of the foregoing arguments, the Arzelá-Ascoli theorem applies and hence $F_{2}$ is compact on $B_{\rho}$. Thus, the hypothesis of Krasnoselskii fixed point theorem [28] is satisfied, which leads to the conclusion that there exists at least one solution on $[0, T]$.

\subsection{Existence Result via Leray-Schauder Nonlinear Alternative}

Here, we prove the existence of solutions for the problem in Equation (1) by means of a nonlinear alternative for single valued maps [29].

Theorem 2. Suppose that $\left(G_{1}\right)$ and the following conditions are satisfied:

$\left(H_{1}\right)$ There exist constants $d_{1}<1 / \Lambda_{1}$ and $d_{2} \geq 0$ such that

$$
|g(t, y)| \leq d_{1}\|y\|+d_{2}, \quad t \in[0, T], y \in \mathbb{R} .
$$


$\left(H_{2}\right)$ There exist a continuous nondecreasing function $\psi:[0, \infty) \rightarrow(0, \infty)$ and a function $p \in C\left([0, T], \mathbb{R}^{+}\right)$ such that

$$
|f(t, y)| \leq p(t) \psi(\|y\|) \text { for each }(t, y) \in[0, T] \times \mathbb{R} .
$$

$\left(H_{3}\right)$ There exists a constant $M>0$ such that

$$
\frac{\left(1-d_{1} \Lambda_{1}\right) M}{d_{2} \Lambda_{1}+\Lambda_{2}\|p\| \psi(M)+\frac{|c|}{|\Omega|}\left[\left|B_{2}\right| \frac{T^{\beta}}{\Gamma(\beta+1)}+\left|A_{2}\right|\right]}>1,
$$

where $\Lambda_{1}$ and $\Lambda_{2}$ are respectively given by Equations (18) and (19).

Then, the BVP in Equation (1) has at least one solution on [0,T].

Proof. We establish in several steps that the operator $F: C([0, T], \mathbb{R}) \rightarrow C([0, T], \mathbb{R})$ defined by Equation (17) is continuous and completely continuous.

Step 1: $F$ is continuous.

Let $\left\{y_{n}\right\}$ be a sequence such that $y_{n} \rightarrow y$ in $C([0, T], \mathbb{R})$. Then,

$$
\begin{aligned}
& \left|F\left(y_{n}\right)(t)-F(y)(t)\right| \\
\leq & J^{\beta}\left|g\left(t, y_{n}(t)\right)-g(t, y(t))\right|+J^{\alpha+\beta}\left|f\left(t, y_{n}(t)\right)-f(t, y(t))\right| \\
& +\frac{T^{\beta}}{|\Omega| \Gamma(\beta+1)}\left\{| B _ { 2 } | \left[\left|a_{1}\right| J^{\beta}\left|g\left(\eta, y_{n}(\eta)\right)-g(\eta, y(\eta))\right|\right.\right. \\
& +\left|b_{1}\right| J^{\beta}\left|g\left(T, y_{n}(T)\right)-g(T, y(T))\right|+\left|a_{1}\right| J^{\alpha+\beta} \mid f\left(\eta, y_{n}(\eta)\right)-f(\eta, y(\eta) \mid \\
& \left.+\left|b_{1}\right| J^{\alpha+\beta}\left|f\left(T, y_{n}(T)\right)-f(T, y(T))\right|\right] \\
& +\left|B_{1}\right|\left[\sum_{i=1}^{n}\left|\lambda_{i}\right|{ }^{\rho_{i}} I_{\theta_{i}, \kappa_{i}}^{\gamma_{i}, \delta_{i}}\left(J^{\beta}\left|g\left(\xi_{i}, y_{n}\left(\xi_{i}\right)\right)-g\left(\xi_{i}, y\left(\xi_{i}\right)\right)\right|+J^{\alpha+\beta}\left|f\left(\xi_{i}, y_{n}\left(\xi_{i}\right)\right)-f\left(\xi_{i}, y\left(\xi_{i}\right)\right)\right|\right)\right. \\
& +\left|a_{2}\right| J^{\beta-p}\left|g\left(\eta, y_{n}(\eta)\right)-g(\eta, y(\eta))\right|+\left|b_{2}\right| J^{\beta-p}\left|g\left(T, y_{n}(T)\right)-g(T, y(T))\right| \\
& \left.+\left|a_{2}\right| J^{\alpha+\beta-p} \mid f\left(\eta, y_{n}(\eta)\right)-f\left(\eta, y(\eta)|+| b_{2}\left|J^{\alpha+\beta-p}\right| f\left(T, y_{n}(T)\right)-f(T, y(T)) \mid\right]\right\} \\
& +\frac{1}{|\Omega|}\left\{| A _ { 1 } | \left[\sum _ { i = 1 } ^ { n } | \lambda _ { i } | | _ { i } I _ { \theta _ { i } , \kappa _ { i } } ^ { \gamma _ { i } , \delta _ { i } } \left(J^{\beta}\left|g\left(\xi_{i}, y_{n}\left(\xi_{i}\right)\right)-g\left(\xi_{i}, y\left(\xi_{i}\right)\right)\right|\right.\right.\right. \\
& \left.+J^{\alpha+\beta}\left|f\left(\xi_{i}, y_{n}\left(\xi_{i}\right)\right)-f\left(\xi_{i}, y\left(\xi_{i}\right)\right)\right|\right) \\
& +\left|a_{2}\right| J^{\beta-p}\left|g\left(\eta, y_{n}(\eta)\right)-g(\eta, y(\eta))\right|+\left|b_{2}\right| J^{\beta-p}\left|g\left(T, y_{n}(T)\right)-g(T, y(T))\right| \\
& +\left|a_{2}\right| J^{\alpha+\beta-p} \mid f\left(\eta, y_{n}(\eta)\right)-f\left(\eta, y(\eta)|+| b_{2}\left|J^{\alpha+\beta-p}\right| f\left(T, y_{n}(T)\right)-f(T, y(T)) \mid\right] \\
& +\left|A_{2}\right|\left[\left|a_{1}\right| J^{\beta}\left|g\left(\eta, y_{n}(\eta)\right)-g(\eta, y(\eta))\right|+\left|b_{1}\right| J^{\beta}\left|g\left(T, y_{n}(T)\right)-g(T, y(T))\right|\right. \\
& \left.+\left|a_{1}\right| J^{\alpha+\beta} \mid f\left(\eta, y_{n}(\eta)\right)-f\left(\eta, y(\eta)|+| b_{1}\left|J^{\alpha+\beta}\right| f\left(T, y_{n}(T)\right)-f(T, y(T)) \mid\right]\right\} \\
\leq & \Lambda_{1}\left\|g\left(\cdot, y_{n}\right)-g(\cdot, y)\right\|+\Lambda{ }_{2}\left\|f\left(\cdot, y_{n}\right)-f(\cdot, y)\right\| .
\end{aligned}
$$

Since $f, g$ are continuous functions, we have

$$
\left\|F\left(y_{n}\right)-F(y)\right\| \leq \Lambda_{1}\left\|g\left(\cdot, y_{n}\right)-g(\cdot, y)\right\|+\Lambda_{2}\left\|f\left(\cdot, y_{n}\right)-f(\cdot, y)\right\| \rightarrow 0 \text { as } n \rightarrow \infty .
$$

Step 2: F maps bounded sets into bounded sets in $C([0, T], \mathbb{R})$. 
Let us show that there exists a positive constant $\tilde{\ell}$ such that $\|F(y)\| \leq \tilde{\ell}$ for each $y \in B_{\theta}=\{y \in$ $C([0, T], \mathbb{R}):\|y\| \leq \theta\}$, where $\theta$ is any positive real number. For each $t \in[0, T]$, it follows by $\left(H_{1}\right)$ and $\left(H_{2}\right)$ that

$$
\begin{aligned}
& |F(y)(t)| \leq J^{\beta}|g(t, y(t))|+J^{\alpha+\beta}|f(t, y(t))|+\frac{T^{\beta}}{|\Omega| \Gamma(\beta+1)}\left\{| B _ { 2 } | \left[|c|+\left|a_{1}\right| J^{\beta}|g(\eta, y(\eta))|\right.\right. \\
& \left.+\left|b_{1}\right| J^{\beta}|g(T, y(T))|+\left|a_{1}\right| J^{\alpha+\beta}|f(\eta, y(\eta))|+\left|b_{1}\right| J^{\alpha+\beta}|f(T, y(T))|\right] \\
& +\left|B_{1}\right|\left[\sum_{i=1}^{n}\left|\lambda_{i}\right|{ }^{\rho_{i}} I_{\theta_{i}, \kappa_{i}}^{\gamma_{i} \delta_{i}}\left(J^{\beta}\left|g\left(\xi_{i}, y\left(\xi_{i}\right)\right)\right|+J^{\alpha+\beta}\left|f\left(\xi_{i}, y\left(\xi_{i}\right)\right)\right|\right)+\left|a_{2}\right| J^{\beta-p}|g(\eta, y(\eta))|\right. \\
& \left.\left.+\left|b_{2}\right| J^{\beta-p}|g(T, x(T))|+\left|a_{2}\right| J^{\alpha+\beta-p}|f(\eta, y(\eta))|+\left|b_{2}\right| J^{\alpha+\beta-p}|f(T, y(T))|\right]\right\} \\
& +\frac{1}{|\Omega|}\left\{| A _ { 1 } | \left[\sum_{i=1}^{n}\left|\lambda_{i}\right| \rho_{i} I_{\theta_{i}, \kappa_{i}}^{\gamma_{i}, \delta_{i}}\left(J^{\beta}\left|g\left(\xi_{i}, y\left(\xi_{i}\right)\right)\right|+J^{\alpha+\beta}\left|f\left(\xi_{i}, y\left(\xi_{i}\right)\right)\right|\right)+\left|a_{2}\right| J^{\beta-p}|g(\eta, y(\eta))|\right.\right. \\
& \left.+\left|b_{2}\right| J^{\beta-p}|g(T, y(T))|+\left|a_{2}\right| J^{\alpha+\beta-p}|f(\eta, y(\eta))|+\left|b_{2}\right| J^{\alpha+\beta-p}|f(T, y(T))|\right] \\
& +\left|A_{2}\right|\left[|c|+\left|a_{1}\right| J^{\beta}|g(\eta, y(\eta))|+\left|b_{1}\right| J^{\beta}|g(T, y(T))|+\left|a_{1}\right| J^{\alpha+\beta}|f(\eta, y(\eta))|\right. \\
& \left.\left.+\left|b_{1}\right| J^{\alpha+\beta}|f(T, y(T))|\right]\right\} \\
& \leq\left(d_{1}\|y\|+d_{2}\right)\left[\frac{T^{\beta}}{\Gamma(\beta+1)}+\frac{T^{\beta}}{|\Omega| \Gamma(\beta+1)}\left\{\left|B_{2}\right| \frac{\left|a_{1}\right| \eta^{\beta}+\left|b_{1}\right| T^{\beta}}{\Gamma(\beta+1)}\right.\right. \\
& \left.+\left|B_{1}\right|\left[\sum_{i=1}^{n}\left|\lambda_{i}\right| \frac{\xi_{i}^{\kappa_{i}+\rho_{i}\left(\theta_{i}+\gamma_{i}\right)+\beta} \Gamma\left(\theta_{i}+\frac{\beta}{\rho_{i}}+1\right)}{\rho_{i}^{\delta_{i}} \Gamma(\beta+1) \Gamma\left(\gamma_{i}+\theta_{i}+\frac{\beta}{\rho_{i}}+1\right)}+\frac{\left|a_{2}\right| \eta^{\beta-p}+\left|b_{2}\right| T^{\beta-p}}{\Gamma(\beta-p+1)}\right]\right\} \\
& +\frac{1}{|\Omega|}\left\{\left|A_{1}\right|\left[\sum_{i=1}^{n}\left|\lambda_{i}\right| \frac{\xi_{i}^{\alpha_{i}+\rho_{i}\left(\theta_{i}+\gamma_{i}\right)+\beta} \Gamma\left(\theta_{i}+\frac{\beta}{\rho_{i}}+1\right)}{\rho_{i}^{\delta_{i}} \Gamma(\beta+1) \Gamma\left(\gamma_{i}+\theta_{i}+\frac{\beta}{\rho_{i}}+1\right)}+\frac{\left|a_{2}\right| \eta^{\beta-p}+\left|b_{2}\right| T^{\beta-p}}{\Gamma(\beta-p+1)}\right]\right. \\
& \left.\left.+\left|A_{2}\right| \frac{\left|a_{1}\right| \eta^{\beta}+\left|b_{1}\right| T^{\beta}}{\Gamma(\beta+1)}\right\}\right] \\
& +\|p\| \psi(\|y\|)\left[\frac{T^{\alpha+\beta}}{\Gamma(\alpha+\beta+1)}+\frac{T^{\beta}}{|\Omega| \Gamma(\beta+1)}\left\{\left|B_{2}\right| \frac{\left|a_{1}\right| \eta^{\alpha+\beta}+\left|b_{1}\right| T^{\alpha+\beta}}{\Gamma(\alpha+\beta+1)}\right.\right. \\
& \left.+\left|B_{1}\right|\left[\sum_{i=1}^{n}\left|\lambda_{i}\right| \frac{\xi_{i}^{\kappa_{i}+\rho_{i}\left(\theta_{i}+\gamma_{i}\right)+\alpha+\beta} \Gamma\left(\theta_{i}+\frac{\alpha+\beta}{\rho_{i}}+1\right)}{\rho_{i}^{\delta_{i}} \Gamma(\alpha+\beta+1) \Gamma\left(\gamma_{i}+\theta_{i}+\frac{\alpha+\beta}{\rho_{i}}+1\right)}+\frac{\left|a_{2}\right| \eta^{\alpha+\beta-p}+\left|b_{2}\right| T^{\alpha+\beta-p}}{\Gamma(\alpha+\beta-p+1)}\right]\right\} \\
& +\frac{1}{|\Omega|}\left\{\left|A_{1}\right|\left[\sum_{i=1}^{n}\left|\lambda_{i}\right| \frac{\xi_{i}^{\alpha_{i}+\rho_{i}\left(\theta_{i}+\gamma_{i}\right)+\alpha+\beta} \Gamma\left(\theta_{i}+\frac{\alpha+\beta}{\rho_{i}}+1\right)}{\rho_{i}^{\delta_{i}} \Gamma(\alpha+\beta+1) \Gamma\left(\gamma_{i}+\theta_{i}+\frac{\alpha+\beta}{\rho_{i}}+1\right)}+\frac{\left|a_{2}\right| \eta^{\alpha+\beta-p}+\left|b_{2}\right| T^{\alpha+\beta-p}}{\Gamma(\alpha+\beta-p+1)}\right]\right. \\
& \left.\left.+\left|A_{2}\right| \frac{\left|a_{1}\right| \eta^{\alpha+\beta}+\left|b_{1}\right| T^{\alpha+\beta}}{\Gamma(\alpha+\beta+1)}\right\}\right]+\frac{|c|}{|\Omega|}\left[\left|B_{2}\right| \frac{T^{\beta}}{\Gamma(\beta+1)}+\left|A_{2}\right|\right] \\
& \leq \Lambda_{1}\left(d_{1}\|y\|+d_{2}\right)+\Lambda_{2}\|p\| \psi(\|y\|)+\frac{|c|}{|\Omega|}\left[\left|B_{2}\right| \frac{T^{\beta}}{\Gamma(\beta+1)}+\left|A_{2}\right|\right] \text {. }
\end{aligned}
$$

Thus,

$$
\|F(y)\| \leq \Lambda_{1}\left(d_{1} \theta+d_{2}\right)+\Lambda_{2}\|p\| \psi(\theta)+\frac{|c|}{|\Omega|}\left[\left|B_{2}\right| \frac{T^{\beta}}{\Gamma(\beta+1)}+\left|A_{2}\right|\right]:=\tilde{\ell} .
$$

Step 3: $F$ maps bounded sets into equicontinuous sets of $C([0, T], \mathbb{R})$.

Let $y \in B_{\theta}$, where $B_{\theta}$ is a bounded set of $C([0, T], \mathbb{R})$. Then, for $t_{1}, t_{2} \in[0, T]$, with $t_{1}<t_{2}$, we have (as in Step 2) 


$$
\begin{aligned}
& \left|F(y)\left(t_{2}\right)-F(y)\left(t_{1}\right)\right| \\
\leq & \frac{d_{1} \theta+d_{2}}{\Gamma(\beta+1)}\left[t_{2}^{\beta}-t_{1}^{\beta}+2\left(t_{2}-t_{1}\right)^{\beta}\right]+\frac{\|p\| \psi(\theta)}{\Gamma(\alpha+\beta+1)}\left[t_{2}^{\alpha+\beta}-t_{1}^{\alpha+\beta}+2\left(t_{2}-t_{1}\right)^{\alpha+\beta}\right] \\
& +\frac{\left.\left|t_{2}^{\beta}-t_{1}^{\beta}\right|\left(d_{1} \theta+d_{2}\right)\right)}{|\Omega| \Gamma(\beta+1)}\left\{\left|B_{2}\right| \frac{\left|a_{1}\right| \eta^{\beta}+\left|b_{1}\right| T^{\beta}}{\Gamma(\beta+1)}+\left|B_{1}\right|\left[\sum_{i=1}^{n}\left|\lambda_{i}\right| \frac{\xi_{i}^{\kappa_{i}+\rho_{i}\left(\theta_{i}+\gamma_{i}\right)+\beta} \Gamma\left(\theta_{i}+\frac{\beta}{\rho_{i}}+1\right)}{\rho_{i}^{\delta_{i}} \Gamma(\beta+1) \Gamma\left(\gamma_{i}+\theta_{i}+\frac{\beta}{\rho_{i}}+1\right)}\right.\right. \\
& \left.\left.+\frac{\left|a_{2}\right| \eta^{\beta-p}+\left|b_{1}\right| T^{\beta-p}}{\Gamma(\alpha+\beta-p+1)}\right]\right\}+\frac{\left|t_{2}^{\beta}-t_{1}^{\beta}\right||| p \| \psi(\theta)}{|\Omega| \Gamma(\beta+1)}\left\{\left|B_{2}\right| \frac{\left|a_{1}\right| \eta^{\alpha+\beta}+\left|b_{1}\right| T^{\alpha+\beta}}{\Gamma(\alpha+\beta+1)}\right. \\
& \left.+\left|B_{1}\right|\left[\sum_{i=1}^{n}\left|\lambda_{i}\right| \frac{\xi_{i}^{\kappa_{i}+\rho_{i}\left(\theta_{i}+\gamma_{i}\right)+\alpha+\beta} \Gamma\left(\theta_{i}+\frac{\alpha+\beta}{\rho_{i}}+1\right)}{\rho_{i}^{\delta_{i}} \Gamma(\alpha+\beta+1) \Gamma\left(\gamma_{i}+\theta_{i}+\frac{\alpha+\beta}{\rho_{i}}+1\right)}+\frac{\left|a_{2}\right| \eta^{\alpha+\beta-p}+\left|b_{2}\right| T^{\alpha+\beta-p}}{\Gamma(\alpha+\beta-p+1)}\right]\right\} \rightarrow 0 \text { as } t_{1} \longrightarrow t_{2},
\end{aligned}
$$

independently of $y \in B_{\theta}$. As argued in the proof of the last theorem, we deduce that the operator $F: C([0, T], \mathbb{R}) \longrightarrow C([0, T], \mathbb{R})$ is continuous and completely continuous.

Step 4: There exists an open set $U \subseteq C([0, T], \mathbb{R})$ with $y \neq \mu F(y)$ for $\mu \in(0,1)$ and $y \in \partial U$.

Let $y \in C([0, T], \mathbb{R})$ be such that $y=\mu F(y)$ for some $0<\mu<1$. Then, for each $t \in[0, T]$, we have

$$
\begin{aligned}
y(t)= & \mu J^{\beta} g(t, y(t))+\mu J^{\alpha+\beta} f(t, y(t))+\mu \frac{t^{\beta}}{\Omega \Gamma(\beta+1)}\left\{-B_{2}\left[c-a_{1} J^{\beta} g(\eta, y(\eta))\right.\right. \\
& \left.-b_{1} J^{\beta} g(T, y(T))-a_{1} J^{\alpha+\beta} f(\eta, y(\eta))-b_{1} J^{\alpha+\beta} f(T, y(T))\right] \\
& -B_{1}\left[\sum_{i=1}^{n} \lambda_{i} \rho_{i} I_{\theta_{i}, \kappa_{i}}^{\gamma_{i}, \delta_{i}}\left(J^{\beta} g\left(\xi_{i}, y\left(\xi_{i}\right)\right)+J^{\alpha+\beta} f\left(\xi_{i}, y\left(\xi_{i}\right)\right)\right)-a_{2} J^{\beta-p} g(\eta, y(\eta))\right. \\
& \left.\left.-b_{2} J^{\beta-p} g(T, y(T))-a_{2} J^{\alpha+\beta-p} f(\eta, y(\eta))-b_{2} J^{\alpha+\beta-p} f(T, y(T))\right]\right\} \\
& +\mu \frac{1}{\Omega}\left\{A _ { 1 } \left[\sum_{i=1}^{n} \lambda_{i} \rho_{i} I_{\theta_{i}, \kappa_{i}}^{\gamma_{i}, \delta_{i}}\left(J^{\beta} g\left(\xi_{i}, y\left(\xi_{i}\right)\right)+J^{\alpha+\beta} f\left(\xi_{i}, y\left(\xi_{i}\right)\right)\right)-a_{2} J^{\beta-p} g(\eta, y(\eta))\right.\right. \\
& \left.-b_{2} J^{\beta-p_{g}} g(T, y(T))-a_{2} J^{\alpha+\beta-p} f(\eta, y(\eta))-b_{2} J^{\alpha+\beta-p} f(T, y(T))\right] \\
& -A_{2}\left[c-a_{1} J^{\beta} g(\eta, y(\eta))-b_{1} J^{\beta} g(T, y(T))-a_{1} J^{\alpha+\beta} f(\eta, y(\eta))\right. \\
& \left.\left.-b_{1} J^{\alpha+\beta} f(T, y(T))\right]\right\} .
\end{aligned}
$$

As argued in Step 2, one can obtain

$$
|y(t)|=|\mu(F y)(t)| \leq \Lambda_{1}\left(d_{1}\|y\|+d_{2}\right)+\Lambda_{2}\|p\| \psi(\|y\|)+\frac{|c|}{|\Omega|}\left[\left|B_{2}\right| \frac{T^{\beta}}{\Gamma(\beta+1)}+\left|A_{2}\right|\right],
$$

which can be expressed as

$$
\frac{\left(1-d_{1} \Lambda_{1}\right)\|y\|}{d_{2} \Lambda_{1}+\Lambda_{2}\|p\| \psi(\|y\|)+\frac{|c|}{|\Omega|}\left[\left|B_{2}\right| \frac{T^{\beta}}{\Gamma(\beta+1)}+\left|A_{2}\right|\right]} \leq 1 .
$$

By Condition $\left(H_{3}\right)$, we can find $M>0$ such that $\|y\| \neq M$. Introduce a set $U=\{y \in C([0, T], \mathbb{R})$ : $\|y\|<\mathrm{M}\}$ and observe that the operator $F: \bar{U} \rightarrow C([0, T], \mathbb{R})$ is continuous and completely continuous. By the choice of $U$, we cannot find a $y \in \partial U$ satisfying $y=\mu F y$ for some $\mu \in(0,1)$. Consequently, we deduce by the nonlinear alternative of Leray-Schauder type [29] that $F$ has a fixed point $y \in \bar{U}$ which is a solution of the problem in Equation (1). This finishes the proof. 
3.3. Uniqueness Result via Banach Fixed Point Theorem

Theorem 3. Assume that:

$\left(H_{4}\right)$ There exists a nonnegative constant $k$ such that

$$
|g(t, x)-g(t, y)| \leq k\|x-y\|, \quad \text { for } t \in[0, T] \text { and every } x, y \in \mathbb{R}
$$

$\left(H_{5}\right)$ there exists $\ell>0$ such that

$$
|f(t, x)-f(t, y)| \leq \ell\|y-x\|, \quad \text { for } t \in[0, T] \text { and every } x, y \in \mathbb{R} \text {. }
$$

Then, there exists a unique solution for the BVP in Equation (1) on the interval $[0, T]$ provided that

$$
k \Lambda_{1}+\ell \Lambda_{2}<1
$$

where $\Lambda_{1}, \Lambda_{2}$ are defined by Equations (18) and (19), respectively.

Proof. In the first step, we prove that $F B_{r} \subset B_{r}$, where the operator $F: C([0, T], \mathbb{R}) \rightarrow C([0, T], \mathbb{R})$ is defined by Equation (17), $B_{r}=\{y \in C([0, T], \mathbb{R}):\|y\| \leq r\}$ and

$$
r \geq \frac{\Lambda_{1} g_{0}+\Lambda_{2} f_{0}+\frac{|c|}{|\Omega|}\left[\left|B_{2}\right| \frac{T^{\beta}}{\Gamma(\beta+1)}+\left|A_{2}\right|\right]}{1-k \Lambda_{1}-\ell \Lambda_{2}}, g_{0}=\sup _{t \in[0, T]}|g(t, 0)|, f_{0}=\sup _{t \in[0, T]}|f(t, 0)|,
$$

where $\Lambda_{1}$ and $\Lambda_{2}$ are, respectively, given by Equations (18) and (19). For $y \in B_{r}$, using $\left(H_{4}\right)$ and $\left(H_{5}\right)$, we get

$$
\begin{aligned}
& |F(y)(t)| \leq\left(k\|y\|+g_{0}\right)\left[\frac{T^{\beta}}{\Gamma(\beta+1)}+\frac{T^{\beta}}{|\Omega| \Gamma(\beta+1)}\left\{\left|B_{2}\right| \frac{\left|a_{1}\right| \eta^{\beta}+\left|b_{1}\right| T^{\beta}}{\Gamma(\beta+1)}\right.\right. \\
& \left.+\left|B_{1}\right|\left[\sum_{i=1}^{n}\left|\lambda_{i}\right| \frac{\xi_{i}^{\kappa_{i}+\rho_{i}\left(\theta_{i}+\gamma_{i}\right)+\beta} \Gamma\left(\theta_{i}+\frac{\beta}{\rho_{i}}+1\right)}{\rho_{i}^{\delta_{i}} \Gamma(\beta+1) \Gamma\left(\gamma_{i}+\theta_{i}+\frac{\beta}{\rho_{i}}+1\right)}+\frac{\left|a_{2}\right| \eta^{\beta-p}+\left|b_{2}\right| T^{\beta-p}}{\Gamma(\beta-p+1)}\right]\right\} \\
& \left.+\frac{1}{|\Omega|}\left\{\left|A_{1}\right|\left[\sum_{i=1}^{n}\left|\lambda_{i}\right| \frac{\xi_{i}^{\kappa_{i}+\rho_{i}\left(\theta_{i}+\gamma_{i}\right)+\beta} \Gamma\left(\theta_{i}+\frac{\beta}{\rho_{i}}+1\right)}{\rho_{i}^{\delta_{i}} \Gamma(\beta+1) \Gamma\left(\gamma_{i}+\theta_{i}+\frac{\beta}{\rho_{i}}+1\right)}+\frac{\left|a_{2}\right| \eta^{\beta-p}+\left|b_{2}\right| T^{\beta-p}}{\Gamma(\beta-p+1)}\right]+\left|A_{2}\right| \frac{\left|a_{1}\right| \eta^{\beta}+\left|b_{1}\right| T^{\beta}}{\Gamma(\beta+1)}\right\}\right] \\
& +\left(\ell\|y\|+f_{0}\right)\left[\frac{T^{\alpha+\beta}}{\Gamma(\alpha+\beta+1)}+\frac{T^{\beta}}{|\Omega| \Gamma(\beta+1)}\left\{\left|B_{2}\right| \frac{\left|a_{1}\right| \eta^{\alpha+\beta}+\left|b_{1}\right| T^{\alpha+\beta}}{\Gamma(\alpha+\beta+1)}\right.\right. \\
& \left.+\left|B_{1}\right|\left[\sum_{i=1}^{n}\left|\lambda_{i}\right| \frac{\xi_{i}^{\kappa_{i}+\rho_{i}\left(\theta_{i}+\gamma_{i}\right)+\alpha+\beta} \Gamma\left(\theta_{i}+\frac{\alpha+\beta}{\rho_{i}}+1\right)}{\rho_{i}^{\delta_{i}} \Gamma(\alpha+\beta+1) \Gamma\left(\gamma_{i}+\theta_{i}+\frac{\alpha+\beta}{\rho_{i}}+1\right)}+\frac{\left|a_{2}\right| \eta^{\alpha+\beta-p}+\left|b_{2}\right| T^{\alpha+\beta-p}}{\Gamma(\alpha+\beta-p+1)}\right]\right\} \\
& +\frac{1}{|\Omega|}\left\{\left|A_{1}\right|\left[\sum_{i=1}^{n}\left|\lambda_{i}\right| \frac{\xi_{i}^{\kappa_{i}+\rho_{i}\left(\theta_{i}+\gamma_{i}\right)+\alpha+\beta} \Gamma\left(\theta_{i}+\frac{\alpha+\beta}{\rho_{i}}+1\right)}{\rho_{i}^{\delta_{i}} \Gamma(\alpha+\beta+1) \Gamma\left(\gamma_{i}+\theta_{i}+\frac{\alpha+\beta}{\rho_{i}}+1\right)}+\frac{\left|a_{2}\right| \eta^{\alpha+\beta-p}+\left|b_{2}\right| T^{\alpha+\beta-p}}{\Gamma(\alpha+\beta-p+1)}\right]\right. \\
& \left.\left.+\left|A_{2}\right| \frac{\left|a_{1}\right| \eta^{\alpha+\beta}+\left|b_{1}\right| T^{\alpha+\beta}}{\Gamma(\alpha+\beta+1)}\right\}\right]+\frac{|c|}{|\Omega|}\left[\left|B_{2}\right| \frac{T^{\beta}}{\Gamma(\beta+1)}+\left|A_{2}\right|\right] \\
& \leq \Lambda_{1}\left(k r+g_{0}\right)+\Lambda_{2}\left(\ell r+f_{0}\right)+\frac{|c|}{|\Omega|}\left[\left|B_{2}\right| \frac{T^{\beta}}{\Gamma(\beta+1)}+\left|A_{2}\right|\right] \leq r,
\end{aligned}
$$


which implies $\|F(y)\| \leq r$ after taking the norm on $[0, T]$. Thus, $F$ maps $B_{r}$ into itself. Next, we show that the operator $F$ is a contraction. For $y, z \in C([0, T], \mathbb{R})$, we obtain by using the notations in Equations (18) and (19) that

$$
\begin{aligned}
& |F(y)(t)-F(z)(t)| \leq k\|y-z\|\left[\frac{T^{\beta}}{\Gamma(\beta+1)}+\frac{T^{\beta}}{|\Omega| \Gamma(\beta+1)}\left\{\left|B_{2}\right| \frac{\left|a_{1}\right| \eta^{\beta}+\left|b_{1}\right| T^{\beta}}{\Gamma(\beta+1)}\right.\right. \\
& \left.+\left|B_{1}\right|\left[\sum_{i=1}^{n}\left|\lambda_{i}\right| \frac{\xi_{i}^{\kappa_{i}+\rho_{i}\left(\theta_{i}+\gamma_{i}\right)+\beta} \Gamma\left(\theta_{i}+\frac{\beta}{\rho_{i}}+1\right)}{\rho_{i}^{\delta_{i}} \Gamma(\beta+1) \Gamma\left(\gamma_{i}+\theta_{i}+\frac{\beta}{\rho_{i}}+1\right)}+\frac{\left|a_{2}\right| \eta^{\beta-p}+\left|b_{2}\right| T^{\beta-p}}{\Gamma(\beta-p+1)}\right]\right\} \\
& +\frac{1}{|\Omega|}\left\{\left|A_{1}\right|\left[\sum_{i=1}^{n}\left|\lambda_{i}\right| \frac{\xi_{i}^{\kappa_{i}+\rho_{i}\left(\theta_{i}+\gamma_{i}\right)+\beta} \Gamma\left(\theta_{i}+\frac{\beta}{\rho_{i}}+1\right)}{\rho_{i}^{\delta_{i}} \Gamma(\beta+1) \Gamma\left(\gamma_{i}+\theta_{i}+\frac{\beta}{\rho_{i}}+1\right)}+\frac{\left|a_{2}\right| \eta^{\beta-p}+\left|b_{2}\right| T^{\beta-p}}{\Gamma(\beta-p+1)}\right]\right. \\
& \left.\left.+\left|A_{2}\right| \frac{\left|a_{1}\right| \eta^{\beta}+\left|b_{1}\right| T^{\beta}}{\Gamma(\beta+1)}\right\}\right] \\
& +\ell\|y-z\|\left[\frac{T^{\alpha+\beta}}{\Gamma(\alpha+\beta+1)}+\frac{T^{\beta}}{|\Omega| \Gamma(\beta+1)}\left\{\left|B_{2}\right| \frac{\left|a_{1}\right| \eta^{\alpha+\beta}+\left|b_{1}\right| T^{\alpha+\beta}}{\Gamma(\alpha+\beta+1)}\right.\right. \\
& \left.+\left|B_{1}\right|\left[\sum_{i=1}^{n}\left|\lambda_{i}\right| \frac{\xi_{i}^{\kappa_{i}+\rho_{i}\left(\theta_{i}+\gamma_{i}\right)+\alpha+\beta} \Gamma\left(\theta_{i}+\frac{\alpha+\beta}{\rho_{i}}+1\right)}{\rho_{i}^{\delta_{i}} \Gamma(\alpha+\beta+1) \Gamma\left(\gamma_{i}+\theta_{i}+\frac{\alpha+\beta}{\rho_{i}}+1\right)}+\frac{\left|a_{2}\right| \eta^{\alpha+\beta-p}+\left|b_{2}\right| T^{\alpha+\beta-p}}{\Gamma(\alpha+\beta-p+1)}\right]\right\} \\
& +\frac{1}{|\Omega|}\left\{\left|A_{1}\right|\left[\sum_{i=1}^{n}\left|\lambda_{i}\right| \frac{\xi_{i}^{\kappa_{i}+\rho_{i}\left(\theta_{i}+\gamma_{i}\right)+\alpha+\beta} \Gamma\left(\theta_{i}+\frac{\alpha+\beta}{\rho_{i}}+1\right)}{\rho_{i}^{\delta_{i}} \Gamma(\alpha+\beta+1) \Gamma\left(\gamma_{i}+\theta_{i}+\frac{\alpha+\beta}{\rho_{i}}+1\right)}+\frac{\left|a_{2}\right| \eta^{\alpha+\beta-p}+\left|b_{2}\right| T^{\alpha+\beta-p}}{\Gamma(\alpha+\beta-p+1)}\right]\right. \\
& \left.\left.+\left|A_{2}\right| \frac{\left|a_{1}\right| \eta^{\alpha+\beta}+\left|b_{1}\right| T^{\alpha+\beta}}{\Gamma(\alpha+\beta+1)}\right\}\right] \\
& =\left(k \Lambda_{1}+\ell \Lambda_{2}\right)\|y-z\| \text {. }
\end{aligned}
$$

Consequently, we get

$$
\|F(y)-F(z)\| \leq\left(k \Lambda_{1}+\ell \Lambda_{2}\right)\|y-z\|,
$$

which implies that $F$ is a contraction by the condition in Equation (21). Hence, we deduce by Banach contraction mapping principle that the operator $F$ has a unique fixed point, which corresponds to a unique solution of the problem in Equation (1) on $[0, T]$. The proof is completed.

\section{Examples}

This section is devoted to the illustration of the results derived in the last section.

Consider the boundary value problem:

$$
\left\{\begin{array}{l}
{ }^{c} D^{2 / 5}\left[{ }^{c} D^{4 / 5} y(t)-g(t, y(t))\right]=f(t, y(t)), t \in[0,2], \\
y(1 / 2)+2 y(2)=1, \\
{ }^{c} D^{1 / 5} y(1 / 2)+{ }^{c} D^{1 / 5} y(2)={ }^{1} I_{0,0}^{1 / 4,1} y(1 / 3)-3{ }^{1 / 2} I_{1 / 3,1 / 7}^{3 / 4,1 / 2} y(1)+2{ }^{3 / 5} I_{0,0}^{1 / 2,1 / 2} y(3 / 2),
\end{array}\right.
$$

where $\alpha=2 / 5, \beta=4 / 5, p=1 / 5, \eta=1 / 2, a_{1}=a_{2}=b_{2}=c=1, b_{1}=2, \gamma_{1}=1 / 4, \delta_{1}=1, \rho_{1} \rightarrow$ $1, \kappa_{1}=\theta_{1}=0, \lambda_{1}=1, \xi_{1}=1 / 3, \gamma_{2}=3 / 4, \delta_{2}=1 / 2, \rho_{2}=1 / 2, \kappa_{2}=1 / 7, \theta_{2}=1 / 3, \lambda_{2}=-3, \xi_{2}=$ $1, \gamma_{3}=\delta_{3}=1 / 2, \rho_{3}=3 / 5, \kappa_{3}=\theta_{3}=0, \lambda_{3}=2, \xi_{3}=3 / 2$, and $g(t, y), f(t, y)$ are fixed below.

Using the given data, we find that $\left|A_{1}\right|=4.355402744,\left|A_{2}\right|=1.232504437,\left|B_{1}\right|=3,\left|B_{2}\right|=$ 0.479215198, $|\Omega|=5.784688499, \Lambda_{1}=16.95703626, \Lambda_{2}=15.33660937$, where $A_{1}, A_{2}, B_{1}, B_{2}, \Omega, \Lambda_{1}$ 
and $\Lambda_{2}$ are, respectively, given by Equations (5)-(9), (18) and (19). To demonstrate the application of Theorem 1, let us take

$$
f(t, y)=\frac{1}{6 \sqrt{900+t^{2}}}\left(\tan ^{-1} y+e^{-t}\right), g(t, y)=\frac{1}{90\left(1+t^{2}\right)} \sin y+\frac{\cos t}{25}
$$

and note that $f(t, y)$ and $g(t, y)$ satisfy the hypothesis of Theorem 1 with $\psi(t)=\frac{1}{90\left(1+t^{2}\right)}+\frac{1}{25}, \phi(t)=$ $\frac{\left(\pi+2 e^{-t}\right)}{12 \sqrt{900+t^{2}}}$ and $k=1 / 90$. In addition, $k \Lambda_{1} \approx 0.1884115140<1$. Thus, the conclusion of Theorem 1 applies to the problem in Equation (22) with $f(t, y)$ and $g(t, y)$ given by Equation (23).

To explain Theorem 2, let

$$
f(t, y)=\frac{1}{6 \sqrt{900+t^{2}}} e^{-t}\left(\tan ^{-1} y+y+\frac{1}{8}\right) \text { and } g(t, y)=\frac{1}{90\left(1+t^{2}\right)} \sin y+\frac{\cos t}{25} .
$$

Obviously, $d_{1}=1 / 90, d_{2}=1 / 25, \psi(\|y\|)=\|y\|+\frac{4 \pi+1}{8}$ and $p(t)=\frac{1}{6 \sqrt{900+t^{2}}} e^{-t}$. By Condition $\left(H_{3}\right)$, we have $M>1.639205778$. Clearly, the hypothesis of Theorem 2 holds true. Thus, it follows by the conclusion of Theorem 2 that the problem in Equation (22), with $f(t, y)$ and $g(t, y)$ given by Equation (24), has at least one solution.

Next, we illustrate Theorem 3 by taking $f(t, y)$ and $g(t, y)$ given by Equation (24). Clearly, Conditions $\left(H_{4}\right)$ and $\left(H_{5}\right)$ hold true with $\ell=1 / 90$ and $k=1 / 90$. In addition, $k \Lambda_{1}+\ell \Lambda_{2} \approx$ $0.3588182848<1$. Clearly, the hypothesis of Theorem 3 is satisfied and hence its conclusion implies the existence of a unique solution of the problem in Equation (22) on $[0,2]$ with $f(t, y)$ and $g(t, y)$ given by Equation (24).

\section{Conclusions}

We have discussed the existence and uniqueness of solutions for a nonlocal multi-term fractional boundary value problem involving generalized integral boundary conditions. Our results are new and enrich the existing literature on multi-term Caputo type fractional differential equations. The work presented in this paper is of quite general nature as the derived results specialize to the ones associated with Riemann-Liouville, Hadamard, Erdélyi-Kober and Katugampola type integral boundary conditions by fixing the parameters involved in generalized integral boundary conditions appropriately (see Remark 1). Furthermore, several new results for multi-term Caputo type fractional differential equations follow by taking the appropriate values of the parameters in the problem in Equation (1). Here are some examples.

- Letting $a_{1}=a_{2}=0$ and $b_{1}=b_{2}=1$ in the boundary conditions of the problem in Equation (1), we get the ones with the boundary conditions:

$$
y(T)=c,{ }^{c} D^{p} y(T)=\sum_{i=1}^{n} \lambda_{i}{ }^{\rho_{i}} I_{\theta_{i}, \kappa_{i}}^{\gamma_{i}, \delta_{i}} y\left(\xi_{i}\right), \eta, \xi_{i} \in(0, T) .
$$

- If we choose $a_{1}=a_{2}=1$ and $b_{1}=b_{2}=0$ for the problem in Equation (1), our results lead to the ones for nonlocal multi-point conditions:

$$
y(\eta)=c,{ }^{c} D^{p} y(\eta)=\sum_{i=1}^{n} \lambda_{i} \rho_{i} I_{\theta_{i}, \kappa_{i}}^{\gamma_{i}} \delta_{i} y\left(\xi_{i}\right), \eta, \xi_{i} \in(0, T)
$$

- Fixing $\lambda_{i}=0(i=1,2,3, \ldots, n), c=0$ and $a_{1}=a_{2}=b_{1}=b_{2}=1$ in the results of this paper, we obtain the ones supplemented with parametric type anti-periodic fractional boundary conditions: $y(\eta)=-y(T),{ }^{c} D^{p} y(\eta)=-{ }^{c} D^{p} y(T), \quad \eta \in(0, T)$, which are indeed new for multi-term Caputo type fractional differential equations. 
Author Contributions: Formal analysis, B.A., M.A., A.A. and S.K.N.

Funding: This research received no external funding.

Acknowledgments: We thank the reviewers for their constructive remarks on our work, which led to the improvement of the original manuscript.

Conflicts of Interest: The authors declare no conflict of interest.

\section{References}

1. Mainardi, F. Some basic problems in continuum and statistical mechanics. In Fractals and Fractional Calculus in Continuum Mechanics; Carpinteri, A., Mainardi, F., Eds.; Springer: Berlin, Germany, 1997; pp. 291-348.

2. Magin, R.L. Fractional Calculus in Bioengineering; Begell House Publishers: Chicago, IL, USA, 2006.

3. Klafter, J.; Lim, S.C.; Metzler, R. (Eds.) Fractional Dynamics in Physics; World Scientific: Singapore, 2011.

4. Yue, Y.; He, L.; Liu, G. Modeling and application of a new nonlinear fractional financial model. J. Appl. Math. 2013, 2013. [CrossRef]

5. Carvalho, A.; Pinto, C.M.A. A delay fractional order model for the co-infection of malaria and HIV / AIDS. Int. J. Dynam. Control 2017, 5, 168-186. [CrossRef]

6. Javidi, M.; Ahmad, B. Dynamic analysis of time fractional order phytoplankton-toxic phytoplankton-zooplankton system. Ecol. Model. 2015, 318, 8-18. [CrossRef]

7. Kilbas, A.A.; Srivastava, H.M.; Trujillo, J.J. Theory and Applications of Fractional Differential Equations; North-Holland Mathematics Studies, 204; Elsevier Science B.V.: Amsterdam, The Netherlands, 2006.

8. Lakshimikantham, V.; Leela, S.; Devi, J.V. Theory of Fractional Dynamic Systems; Cambridge Academic Publishers: Cambridge, UK, 2009.

9. Diethelm, K. The Analysis of Fractional Differential Equations. An Application-oriented Exposition Using Differential Operators of Caputo Type; Lecture Notes in Mathematics 2004; Springer: Berlin, Germany, 2010.

10. Ahmad, B.; Alsaedi, A.; Ntouyas, S.K.; Tariboon, J. Hadamard-Type Fractional Differential Equations, Inclusions and Inequalities; Springer: Cham, Switzerland, 2017.

11. Glushak, A.V. On an inverse problem for a fractional-order abstract differential equation. Math. Notes 2010, 87, 654-662. [CrossRef]

12. Graef, J.R.; Kong, L.; Kong, Q. Application of the mixed monotone operator method to fractional boundary value problems. Fract. Calc. Differ. Calc. 2011, 2, 554-567. [CrossRef]

13. Bai, Z.B.; Sun, W. Existence and multiplicity of positive solutions for singular fractional boundary value problems. Comput. Math. Appl. 2012, 63, 1369-1381. [CrossRef]

14. Ahmad, B.; Ntouyas, S.K. A higher-order nonlocal three-point boundary value problem of sequential fractional differential equations. Miscolc Math. Notes 2014, 15, 265-278. [CrossRef]

15. Li, C.-G.; Kostic, M.; Li, M. Abstract multi-term fractional differential equations. Kragujevac J. Math. 2014, 38, 51-71. [CrossRef]

16. Peng, L.; Zhou, Y. Bifurcation from interval and positive solutions of the three-point boundary value problem for fractional differential equations. Appl. Math. Comput. 2015, 257, 458-466. [CrossRef]

17. Zhang, L.; Ahmad, B.; Wang, G. Successive iterations for positive extremal solutions of nonlinear fractional differential equations on a half line. Bull. Aust. Math. Soc. 2015, 91, 116-128. [CrossRef]

18. Liu, Y. Boundary value problems of singular multi-term fractional differential equations with impulse effects. Math. Nachr. 2016, 289, 1526-1547. [CrossRef]

19. Ntouyas, S.K.; Tariboon, J.; Thaiprayoon, C. Nonlocal boundary value problems for Riemann-Liouville fractional differential inclusions with Hadamard fractional integral boundary conditions. Taiwanese J. Math. 2016, 20, 91-107. [CrossRef]

20. Ahmad, B.; Ntouyas, S.K. Existence results for fractional differential inclusions with Erdelyi-Kober fractional integral conditions. An. Ştiinţ. Univ. Ovidius Constanţa Ser. Mat. 2017, 25, 5-24. [CrossRef]

21. Henderson, J.; Luca, R. Existence of nonnegative solutions for a fractional integro-differential equation. Results Math. 2017, 72, 747-763. [CrossRef]

22. Stanek, S. Periodic problem for two-term fractional differential equations. Fract. Calc. Appl. Anal. 2017, 20, 662-678. [CrossRef] 
23. Ahmad, B.; Matar, M.M.; El-Salmy, O.M. Existence of solutions and Ulam stability for Caputo type sequential fractional differential equations of order $\alpha \in(2,3)$. Inter. J. Anal. Appl. 2017, 15, 86-101.

24. Sayevand, K.; Pichaghchi, K. Efficient algorithms for analyzing the singularly perturbed boundary value problems of fractional order. Commun. Nonlinear Sci. Numer. Simul. 2018, 57, 136-168. [CrossRef]

25. Nyamoradi, N.; Zhou, Y. Multiple solutions for a nonlinear fractional boundary value problems via variational methods. Fixed Point Theory 2016, 17, 111-122.

26. Ahmad, B.; Luca, R. Existence of solutions for a sequential fractional integro-differential system with coupled integral boundary conditions. Chaos Solitons Fractals 2017, 104, 378-388. [CrossRef]

27. Katugampola, U.N. New fractional integral unifying six existing fractional integrals. arXiv 2016, arXiv:1612.08596v1.

28. Krasnoselskii, M.A. Two remarks on the method of successive approximations. Uspekhi Mat. Nauk 1955, 10, 123-127.

29. Granas, A.; Dugundji, J. Fixed Point Theory; Springer: New York, NY, USA, 2003.

(C) 2019 by the authors. Licensee MDPI, Basel, Switzerland. This article is an open access article distributed under the terms and conditions of the Creative Commons Attribution (CC BY) license (http:/ / creativecommons.org/licenses/by/4.0/). 